\title{
The Sun Shadow of Positioning Technology \\ JiXiang Zhou
}

\author{
North China Electric Power University, Baoding ,China \\ 2292686832@qq.com
}

\begin{abstract}
Keywords: The solar height angle; Curve fitting; Residual analysis
\end{abstract}
\begin{abstract}
We will have the date and time and put it into the model of the relationship between the solar height angle and local time, to obtain the length of the shadow of the functional relationship between theoretical value and observed latitude. We divide the length of the shadow of two adjacent times to eliminate the length of the pole. We minimize the error between the theoretical and actual values of the ratio of the length of the adjacent shadow to the objective function, and then use the search method to determine the minimum error of the straight rod location and local time.
\end{abstract}

\section{Introduction}

In the case of known changes in the length of the shadow, we establish the model to determine the location of the straight bar, that is, to determine the latitude and longitude here. Known shooting date and shooting time in Beijing, we can use the sun height angle and the local time relationship model, to obtain the sun height angle changes [1-2].

As the straight rod length is not given, we need to convert a shadow change to a series of data that only the sun's height angle $\theta$ is related by $l=L /(\tan \theta)$.We use the solar elevation angle and the local time relationship model to find the sun height angle change. Then we fit the two quantities to find a number of possible locations.

\section{The Establishment and Solution of Model}

The assumptions and justifications is (1)The earth is rounded around the sun; (2)The sun turns to uniform; (3)The sun's horns remain the same in one day; (4)365 days a year.

We can get the solar elevation angle change on April 18, 2015 through the solar altitude angle and the local time relationship model [3]:

$$
\theta=\frac{\pi}{2}-\arccos \left(-\cos \frac{y \times \pi}{180} \cos \frac{(t-12) \times \pi}{12} \cos \delta-\sin \frac{y \times \pi}{180} \sin \delta\right)
$$

The relationship between Beijing time $t_{0}$ and local time $t$ is [4]:

$$
t=t_{0}+\frac{x-120}{15}
$$

The solar elevation angle changes to:

$$
\theta=\frac{\pi}{2}-\arccos \left(-\cos \frac{y \times \pi}{180} \cos \frac{\left(t_{0}+\frac{x}{15}-4\right) \times \pi}{12} \cos \delta-\sin \frac{y \times \pi}{180} \sin \delta\right)
$$

We use the MATLAB fitting curve to satisfy the following conditions at the same time [5].

$$
\min \sum_{i=2}^{m}\left(\frac{\tan \theta_{i}}{\tan \theta_{i-1}}-\frac{\tan \theta_{i}^{*}}{\tan \theta_{i-1}^{*}}\right)^{2} \quad\left\{\begin{array}{l}
0^{\circ} \leq x \leq 360^{\circ} \\
s t \quad-90^{\circ} \leq y \leq 90^{\circ} \\
2 \leq i \leq m
\end{array}\right.
$$

Where $\tan \theta_{i}^{*} / \tan \theta_{i-1}^{*}$ is the ratio of the solar height angle to the local time relationship model obtained by the corresponding time sun height angle tangent ratio.

We have 1 degree for the step, from 0 degrees to the longitude 180, latitude 90 to latitude 90 search, require all variance of the results of less than $1 \times 10^{-6}$, to meet the requirements of a total 
of twelve points, the latitude and longitude coordinates are as follows:

Table 1 The place where the rod may be

\begin{tabular}{lllllllllllll}
\hline & 1 & 2 & 3 & 4 & 5 & 6 & 7 & 8 & 9 & 10 & 11 & 12 \\
\hline longitude & 102 & 104 & 111 & 110 & 108 & 109 & 107 & 108 & 106 & 104 & 102 & 99 \\
latitude & -4 & -3 & 17 & 18 & 19 & 19 & 20 & 20 & 21 & 22 & 23 & 24
\end{tabular}

We sort out these data and found that these points were concentrated in three areas, namely 102 to 104 degrees east longitude, 3 to 4 degrees south latitude, 106 to 111 degrees east longitude, 18 to 20 degrees north latitude, 99 degrees to 104 degrees east longitude, latitude 21 To 24 degrees. We then search for these three regions to find the actual collection of sun shadow data with a good coordinate position.

From east to 100 degrees to 105 degrees, latitude 2 to 5 degrees, to step distance of 0.01 degrees search results as shown in Fig.1. From east to 105 degrees to 112 degrees, latitude 17 to 21 degrees, to step distance of 0.01 degrees search knot Fig.2. From east to 97 degrees to 105 degrees, latitude 20 to 25 degrees, to step distance of 0.01 degrees search results as shown in Fig.3.

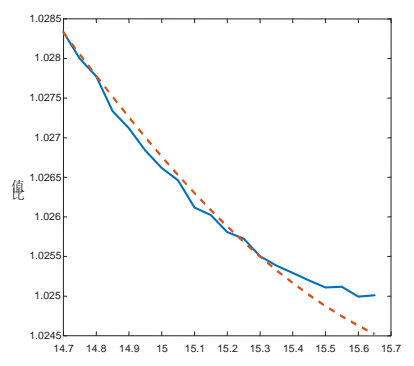

Fig.1 Result

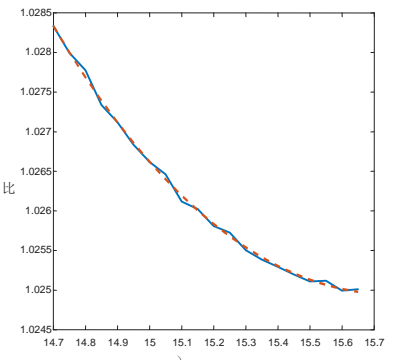

Fig.2 Result

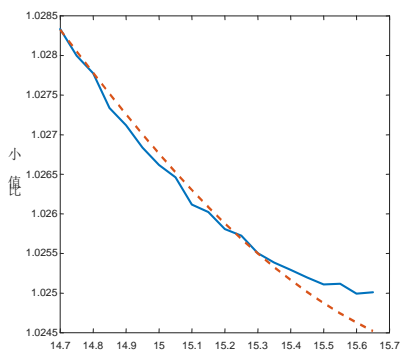

Fig.3 Result

In the three possible areas found in the three precise coordinates of the following table.

Table 2 The exact position of the straight bar

\begin{tabular}{cccc}
\hline & Position one & Position two & Position three \\
\hline longitude & $103.48^{\circ} \mathrm{E}$ & $108.54^{\circ} \mathrm{E}$ & $102^{\circ} \mathrm{E}$ \\
latitude & $3.29^{\circ} \mathrm{S}$ & $19.19^{\circ} \mathrm{N}$ & $23.03^{\circ} \mathrm{N}$
\end{tabular}

We perform residual and variance analysis on these three coordinates, and then use MATLAB to draw the coordinates of the three coordinates of the map. Residual analysis is shown as Fig.4.

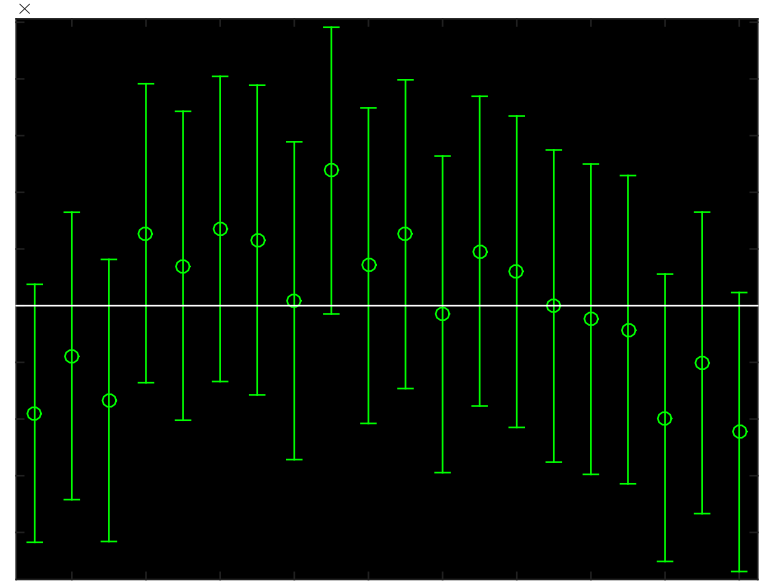



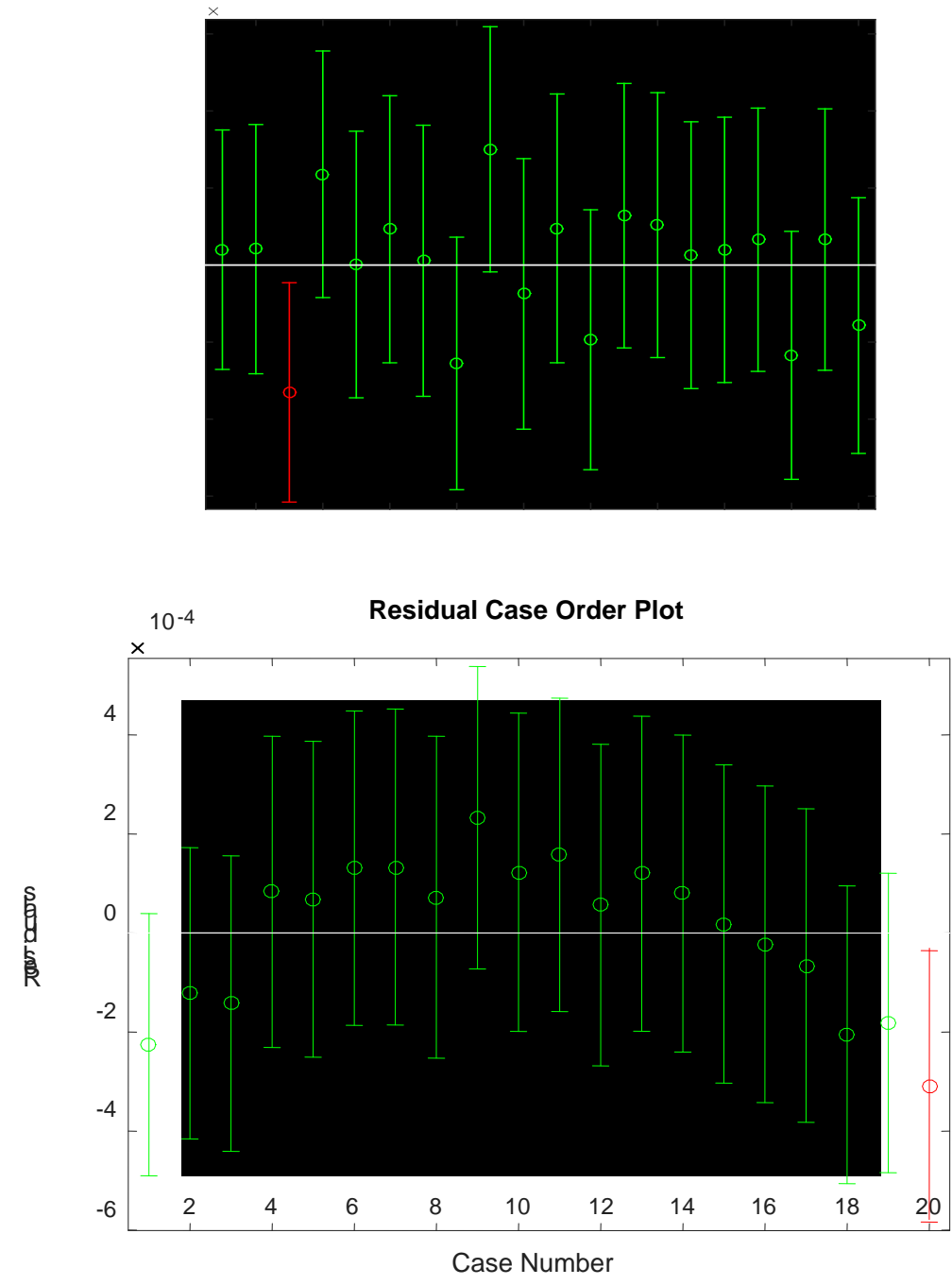

Fig.4 Residual analysis

\section{Summary}

From the three-coordinate position of the three coordinate positions and the residual map, we find that there are abnormal values in the residuals of the coordinate positions of two or three, which may be due to the measurement of the vertex coordinates of the shadow Of the errors in our analysis need to eliminate these outliers. It can be seen from the figure, the residual position of the three coordinates of the residuals in the confidence interval.

It can be found that the curve of the coordinate position is the best, the residual confidence interval is very small, the variance and also reach the order of A, we can see that the credibility of the coordinate position two is very high. The coordinates of the coordinates of the first and third of the curve fit slightly worse than the point two, but also belong to the fitting situation is good, the location of a residual confidence interval is less than the location of two, the difference between the two and the situation is not significant, the reliability of the analysis high.

\section{Reference}

[1] Wu L, Cao X C, Foroosh H. Camera calibration and geo-location estimation from two shadow trajectories [J]. Computer Vision and Image Understanding, 2010, 114: 915-927.

[2]Junejo l N, Foroosh H. UPS coordinates estimation and camera calibration from solar shadows[J].Computer Vision and Image Understanding, 2010， 114:991-1003. 
[3]Zhijie Cai. The sun shadow of positioning technology. Mathematical Modeling and Its Application [M].2015.

[4]Shoukui Si, Zhaoliang Sun. Mathematical modeling algorithm and application.[M].

Second edition. Beijing: National Defense Industry Press.2015.

[5]http://haike.haidu.com/link?url=HFytiOaMbII\}90ue0a8Vz14C6oZshe0-kcxik6VMA8zmjTFwsx QQg3Sar3eoovpWEfZJMwIeX9 xPW C2goA-eScq, 2015. 9. 12 